\title{
The optimization of profiled diffusers
}

Trevor J. Cox

School of ESD, South Bank University, 103 Borough Road, London SE1 OAA, United Kingdom

(Received 11 August 1993; revised 12 December 1994; accepted 20 December 1994)

\begin{abstract}
Methods have been developed to produce profiled diffusers that create a large amount of diffusion. The methods are iterative and required the development of a new parameter to measure diffusion. Achieving scattering independent of angle has been attempted over a wide bandwidth. The methods are also applicable to other diffusion criteria. The diffusers consists of a series of wells of the same width but of different depths similar to Schroeder diffusers. Applications include concert halls, theatres, and studio monitor rooms. The new diffusers have been shown to create better, more uniform diffusion than the previous designs of Schroeder. This is due to the new designs being reliant on accurate boundary element prediction methods rather than more approximate techniques.
\end{abstract}

PACS numbers: 43.55.Br, 43.20.Fn

\section{INTRODUCTION}

Profiled diffusers based on wells were introduced by Schroeder; ${ }^{1}$ an example of one is shown in Fig. 1(a). The one-dimensional form of these diffusers consists of a series of wells, of the same width, but of different depths separated by thin fins. The diffusers are long and uniform in one direction so the scattering is mainly in one plane dominated by the effects of the well depth sequence.

Schroeder diffusers are based on the following concepts. When sound is incident on the diffusers plane waves propagate up and down each well. The waves then radiate from the wells and create an interference pattern. The relative phases of these radiating waves can be altered by changing the well depths. By choosing an appropriate well depth sequence, a particular desired interference pattern can be obtained. To choose a suitable depth sequence Schroeder exploited the fact that the Fourier transform of a "surface" approximately gives the far-field diffracted pressure distribution. If the quadratic residue sequence is used to determine the well depths, the Fourier transform of the surface is constant. Hence such a diffuser should produce optimum diffusion. Due to approximations necessary in the design theory, however, the true scattering is only ever an approximation to optimum diffusion. More detailed discussions of the design of Schroeder diffusers can be found in Refs. 1-4.

In previous work on diffusers used in auditoria, ${ }^{4}$ a variety of methods for predicting the scattering from Schroeder diffusers have been developed and tested. As shall be shown later, it is possible to use these methods in an iterative manner to produce diffusers which are better than those based on the quadratic residue sequence.

It is also possible to apply this iterative method to diffusers of different construction to Schroeder diffusers. This has been demonstrated by optimizing one other type of diffuser which can be described as a Schroeder diffuser without fins. This type of surface will be referred to as a stepped diffuser; an example is shown in Fig. 1(b). Being able to construct diffusers without these fins is an advantage to acousticians. These fins add to construction costs and are one of the most likely sources of absorption.

An investigation into the optimization of profiled diffus- ers has been made once before. ${ }^{5}$ This was restricted to narrow deep wells and a single frequency. The narrow deep wells would not necessarily be appropriate for use in auditoria where minimizing absorption is essential, and wideband diffusion is required. Furthermore, the optimization processes and evaluation of the scattering relied on a relatively simple prediction theory. In this paper all the resulting scattering is evaluated using an accurate boundary element method. ${ }^{4}$ This has also been used for the optimization processes when computation time was not excessively long.

\section{PREDICTION METHODS}

To predict the scattering from the diffusers, the fact that they are uniform and long in one direction was exploited. This allowed two-dimensional prediction methods to be applied to a cross section through the diffuser. These methods produce good predictions of the diffracted pressure distribution, but not the overall magnitude. ${ }^{6}$ This is not a problem for this investigation where the scattered pressure distribution only is required.

Prediction methods based on two boundary element methods (BEMs) formulated from the Helmholtz-Kirchhoff integral equation have been used. These were the standard BEM and thin panel BEM. Brief details are given below. This is followed by a description of how the methods are applied to the various diffusers.

\section{A. Helmholtz-Kirchhoff integral equation}

The prediction methods were based on the single frequency form of the Helmholtz-Kirchhoff integral equation and its normal derivative. For a single source at $\mathbf{r}_{0}$ and a surface $S$, they give the pressure $P(\mathbf{r})$ and its derivative as ${ }^{7}$

$$
\begin{aligned}
\alpha P(\mathbf{r})= & \int_{S}\left(\frac{\partial G\left(\mathbf{r}, \mathbf{r}_{s}\right)}{\partial \mathbf{n}\left(\mathbf{r}_{s}\right)}-i k \beta\left(\mathbf{r}_{s}\right) G\left(\mathbf{r}, \mathbf{r}_{s}\right)\right) P\left(\mathbf{r}_{s}\right) d S \\
& +P_{i}\left(\mathbf{r}, \mathbf{r}_{0}\right),
\end{aligned}
$$




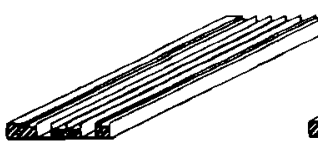

(a)

Cross sections

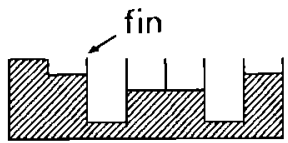

(a)

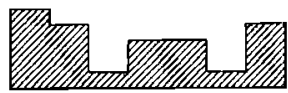

(b)
FIG. 1. The diffusers tested: (a) Schroeder-type diffuser, (b) stepped diffuser without fins.

$$
\begin{aligned}
\alpha \frac{\partial P(\mathbf{r})}{\partial \mathbf{n}(\mathbf{r})}= & \int_{S}\left(\frac{\partial^{2} G\left(\mathbf{r}, \mathbf{r}_{s}\right)}{\partial \mathbf{n}(\mathbf{r}) \mathbf{n}\left(\mathbf{r}_{s}\right)}\right. \\
& \left.-i k \beta\left(\mathbf{r}_{s}\right) \frac{\partial G\left(\mathbf{r}, \mathbf{r}_{s}\right)}{\partial \mathbf{n}(\mathbf{r})}\right) P\left(\mathbf{r}_{s}\right) d S+\frac{\partial P_{i}\left(\mathbf{r}, \mathbf{r}_{0}\right)}{\partial \mathbf{n}(\mathbf{r})}
\end{aligned}
$$

where $P_{i}\left(\mathbf{r}, \mathbf{r}_{0}\right)$ is the sound pressure direct from the source, $G\left(\mathbf{r}, \mathbf{r}_{s}\right)$ is the appropriate Green's function, $\beta\left(\mathbf{r}_{s}\right)$ is the locally reacting surface admittance, and $\mathbf{n}\left(\mathbf{r}_{s}\right)$ is the unit vector normal to the surface, pointing out of the surface. $\alpha$ can have values of $0,1 / 2$, or 1 depending, respectively, on whether the point $\mathbf{r}$ lies within, on the surface of, or external to the reflecting object. Figure 2 shows definitions of the vectors used.

The Green's function was taken to be the standard twodimensional form for free space:

$$
G\left(\mathbf{r}, \mathbf{r}_{s}\right)=-(i / 4) H_{0}^{(1)}\left(k\left|\mathbf{r}-\mathbf{r}_{s}\right|\right),
$$

where $H_{\delta}^{(1)}(x)$ is the Hankel function of order 0 . This was evaluated using a standard polynomial approximation. ${ }^{8}$

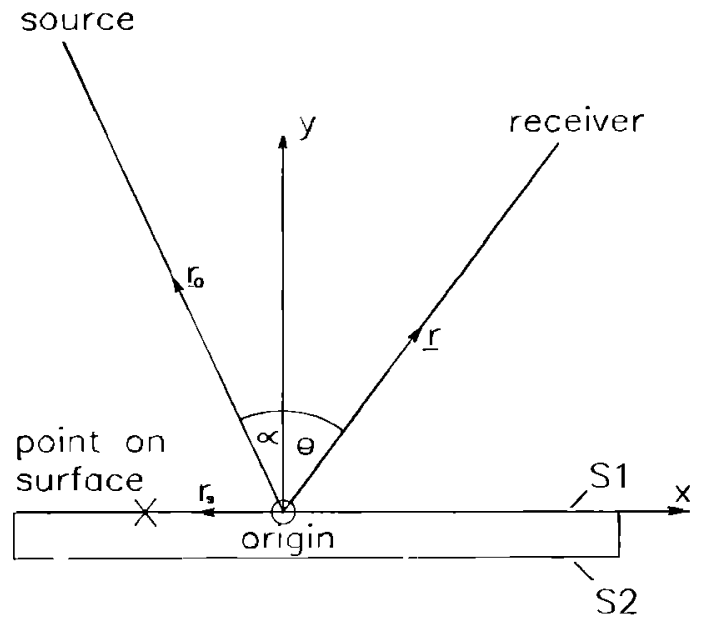

FIG. 2. Geometry used in prediction methods.
Where possible symmetry was exploited. In that case only half the diffuser is modeled and the principle of image sources used; this decreases prediction times. From this, the Green's function is altered to include image sources:

$$
G\left(\mathbf{r}, \mathbf{r}_{s}\right)=-(i / 4)\left[H_{0}^{(1)}\left(k\left|\mathbf{r}-\mathbf{r}_{s}\right|\right)+H_{0}^{(1)}\left(k\left|\mathbf{r}-\mathbf{r}_{s}^{\prime}\right|\right)\right],
$$

where $\mathbf{r}_{s}^{\prime}$ is the position of the image source.

The solution of the Helmholtz-Kirchhoff integral equation in the thin panel limit was also used. Consider the special case of a rigid panel whose thickness reduces to be infinitely thin. Figure 2 shall be used as an example of such a body. The following nomenclature is used: a subscript 1 or 2 is introduced for the front and back surfaces, and $n\left(r_{s 1}\right)$ is written as $\mathbf{n}$. It can then be shown ${ }^{7}$ that Eqs. (1) and (2) become

$$
\begin{aligned}
& 0=\int_{S 1}\left[P\left(\mathbf{r}_{s 1}\right)-\dot{\left.P\left(\mathbf{r}_{s 2}\right)\right]} \frac{\partial^{2} G\left(\mathbf{r}, \mathbf{r}_{s}\right)}{\partial \mathbf{n}(\mathbf{r}) \partial \mathbf{n}\left(\mathbf{r}_{s}\right)} d S\right. \\
& +\frac{\partial P_{i}\left(\mathbf{r}, \mathbf{r}_{0}\right)}{\partial \mathbf{n}(\mathbf{r})} \\
& P(\mathbf{r})=\int_{S 1}\left[P\left(\mathbf{r}_{s 1}\right)-P\left(\mathbf{r}_{s 2}\right)\right] \frac{\partial G\left(\mathbf{r}, \mathbf{r}_{s}\right)}{\partial \mathbf{n}\left(\mathbf{r}_{s}\right)} d S+P_{i}\left(\mathbf{r}, \mathbf{r}_{0}\right),
\end{aligned}
$$

where Eq. (5) is valid only when $r$ lies on the surface; Eq. (6) is for external receiver positions. The surface integral is now only carried out on one side of the thin panel as the equations are in terms of the pressure difference across the panels.

Equations (1)-(6) form the basis of the boundary element methods. A BEM solution for the pressure at $r$ can be found for a surface of known geometry and known local reacting surface admittance. ${ }^{7}$ The general solution method is to break the diffuser down into a set of surface elements. Across each element it is assumed that the pressure and admittance are constant. A set of simultaneous equations is then set up and solved to obtain the surface pressure on each of these elements. Once the surface pressures are known Eq. (1) or (6) is applied to obtain the external point pressure.

\section{B. Predicting the scattering from diffusers with fins}

For diffusers with fins such as the Schroeder diffusers, two methods ${ }^{4}$ have been used. The first is based on the thin panel BEM, the second on the standard BEM.

\section{Thin panel BEM}

When the diffuser is broken down into a set of thin panel elements, all surfaces can be modeled including the thin fins. The method uses Eqs. (5) and (6). The complete enclosure of the diffuser with a zero velocity boundary condition ensures that the pressures on the rear of the nonfin elements and within the diffuser's body are zero. Obtaining the external point pressures from these integral equations is an established technique. ${ }^{9}$ There was a possibility of nonunique or no solutions at critical frequencies.

To check for unique solutions, the predictions using this method were compared to those produced by the standard BEM described below. As the thin panel BEM relies on the derivative of the Helmholtz-Kirchhoff integral equation, 
whereas the standard BEM relies on the normal HelmholtzKirchhoff integral equation, it is highly unlikely that these methods would have the same critical frequencies. If the results were similar it was assumed that the solutions were accurate and unique.

The thin panel BEM has the capability to produce accurate results even when plane-wave propagation within the wells breaks down. (Then methods have to be employed to ensure unique solutions, ${ }^{10}$ as the standard BEM becomes inaccurate and so can not be used for comparison.) Prediction times can become prohibitively long for large complicated surfaces. ${ }^{4}$

\section{Standard BEM}

It is not possible to handle the thin fins in the numerical solution using the standard BEM, and so an approximate representation of the diffuser is required. The diffuser is represented by a box with a variable admittance on the front face. These front face admittances are calculated assuming that the plane waves propagate up and down the wells inducing a phase change with no absorption. For this to be true, hard surfaces, local reaction, and small radiation admittance are assumed. Such an approximate representation is a common first step when predicting the scattering from these diffusers.

Once the diffuser has been represented by this simpler geometry, it is then possible to solve the system using a standard BEM based on Eq. (1). The CHIEF method can be used to confirm unique solutions. ${ }^{11}$ During the optimization process, however, the CHIEF method was neglected to decrease computation time.

The standard BEM is reasonably accurate, but because of the approximations when representing the diffuser geometry, not as accurate as the thin panel BEM. ${ }^{4}$ The standard BEM is, however, much faster for two reasons. First, the number of elements required to represent the diffuser is much smaller. Second, because the approximate box geometry of the diffuser stays the same during the optimization process, it is only necessary to calculate the surface integrals which determine the element-element interactions once.

\section{Predicting the scattering from stepped diffusers}

Two prediction methods were also available for the stepped diffuser. In this case, however, both utilized the standard BEM.

(i) The first method exactly models the diffuser's surface-this is possible because no fins are present. Predicting the scattering from an arbitrary shaped rigid surface by BEMs is a well established, accurate technique.

(ii) The second method used an approximate representation of the diffuser, utilizing a simple phase change local reacting admittance as was used for the diffusers with fins. Where the surface geometry was such that there was a well in the surface, this was replaced by a plane surface with a phase change admittance at the top. This reduced the number of surface elements by up to $\sim 10 \%$ and so reduced calculation time. Tests showed this to induce a small but acceptable error.
For both (i) and (ii) the CHIEF ${ }^{11}$ method was available to ensure unique solutions. This was not used during the optimization process, only during the latter evaluation of the scattering.

\section{LIMITATIONS OF SCHROEDER'S DESIGN THEORY}

Schroeder's original theory can be derived from the Helmholtz-Kirchhoff integral equation by a method analogous to Fraunhofer diffraction in optics. First, Kirchhoff's boundary conditions ${ }^{9}$ have to be applied to Eq. (1). These boundary conditions give values for the surface pressure on the front of the diffuser as $\left[1+R\left(\mathbf{r}_{s}\right)\right] P_{i}\left(\mathbf{r}_{s}\right)$, where $R\left(\mathbf{r}_{s}\right)$ is the reflection factor and $P_{i}\left(\mathbf{r}_{s}\right)$ the incident pressure. The pressures on the back and sides are neglected. This leads to a scattered pressure $P_{s}(\mathbf{r})$ of

$$
\begin{aligned}
P_{s}(\mathbf{r})= & A \int_{S 1}\left[1+R\left(\mathbf{r}_{s}\right)\right] G\left(\mathbf{r}_{s}, \mathbf{r}_{0}\right) \\
& \times\left(\frac{\partial G\left(\mathbf{r}, \mathbf{r}_{s}\right)}{\partial \mathbf{n}\left(\mathbf{r}_{s}\right)}-i k \beta\left(\mathbf{r}_{s}\right) G\left(\mathbf{r}, \mathbf{r}_{s}\right)\right) d S .
\end{aligned}
$$

In terms of well depths, the admittance and reflection factor are given by

$$
\beta\left(\mathbf{r}_{s}\right)=-i \tan \left[k d\left(\mathbf{r}_{s}\right)\right], \quad R\left(\mathbf{r}_{s}\right)=\frac{1-\beta\left(\mathbf{r}_{s}\right)}{1+\beta\left(\mathbf{r}_{s}\right)},
$$

where $d\left(\mathbf{r}_{s}\right)$ is the well depth at $\mathbf{r}_{s}$. An assumption will be made that the source and receiver distances are large compared to wavelength. Then the large argument asymptotic expansion for the Hankel function can be used; this is ${ }^{8}$

$$
H_{\nu}^{(1)}(x) \approx \sqrt{2 / \pi x} e^{i(x-\nu \pi / 2-\pi / 4)} .
$$

Substituting the appropriate Green's function into Eq. (7), neglecting terms in $1 / r^{2}$, and using far-field assumptions yields

$$
\begin{aligned}
P_{s}(\mathbf{r})= & \frac{-A[\cos (\theta)+\cos (\alpha)]}{8 \pi \sqrt{\left|\mathbf{r} \| \mathbf{r}_{0}\right|}} \\
& \times \int_{S 1} R\left(\mathbf{r}_{s}\right) e^{-i k x_{s}[\sin (\alpha)+\sin (\theta)]} d x_{s},
\end{aligned}
$$

where $A$ gives a measure of the source strength, $x_{s}$ is the $x$ component of the vector $r_{s}$, and $\alpha$ and $\theta$ are the source and receiver angles to the surface's normal.

Equation (10) and its derivation show the limitations behind Schroeder's original design and the reasons why optimum diffusion is not produced. This equation does represent a Fourier transform of the surface, except for terms in $\sin (\theta)+\sin (\alpha)$. Only without these terms will the Fraunhofer theory yield constant scattering whatever the receiver angle. The are two further major approximations: (i) The quadratic residue sequence should be repeated to infinity. The truncation necessary for realistic finite diffusers will affect the production of optimum diffusion because of edge effects. (ii) Equation (10) is only an approximate formulation. For these reasons Schroeder diffusers fail to produce optimum diffusion. 


\section{OPTIMUM DIFFUSER DESIGN}

The new process to produce optimum diffusers was based on an iterative process:

(1) A diffuser was constructed with a randomly determined depth sequence.

(2) The pressure scattered from the diffuser was calculated using one of the BEMs.

(3) The scattered pressure distribution was used to calculate a parameter which measured the degree of diffusion (the parameter is described below).

(4) The well depths were altered according to standard minimization techniques.

(5) Steps (2)-(4) were repeated until a minimum in the diffusion parameter was found indicating optimum diffusion.

Two standard minimization techniques were used: a simple but robust downhill simplex method ${ }^{12}$ and a more sophisticated quasi-Newton method. ${ }^{13}$ Both methods only use the diffusion parameter's value, its derivative not being known. It was necessary to run the optimization process many times with different starting conditions. The reason for this is that the minimization was being carried out within bounded space. The space held many local minima within which the minimization routines could become trapped. The solutions presented here are the best found from many attempts of the iteration process. This does not exclude the possibility that from some particular starting point yet untried there might be a better minimum achievable.

\section{A. Diffusion parameter}

For such an optimization process to work a suitable diffusion parameter had to be developed. This parameter has to reduce the large number of results for pressure versus receiver position to a single measure of diffusion. A measure based on a $95 \%$ confidence limit standard error $\epsilon$ was deemed appropriate:

$$
\epsilon=\frac{20}{\ln (10) \bar{I}_{\theta}} \sqrt{\sum_{\theta=-90}^{90}\left(I_{\theta}-\bar{I}_{\theta}\right)^{2} /[n(n-1)]}
$$

where $I_{\theta}$ is the intensity at some angle of reflection $\theta, \bar{I}_{\theta}$ is the average value over $180^{\circ}$, and $n$ is the number of samples in $180^{\circ}$. Using this standard error formulation to measure diffusion is similar to the concept suggested by Schroeder ${ }^{1}$ which was to use "the standard deviation in decibels of the energy fluxes." Equation (11) calculates the standard error via the intensities and so penalizes nonuniform diffusion more than if decibel values were used. The formulation converts the final value to decibels and represents a $95 \%$ confidence limit calculation.

A small value of the standard error indicated good uniform diffusion, a large value poor diffusion. The ideal of complete uniform scattering into all angles would have a diffusion parameter of 0 . If another scattering distribution other than uniform is required, the intensities can be weighted by the inverse of the desired function before the calculation of the standard error. The standard error can be

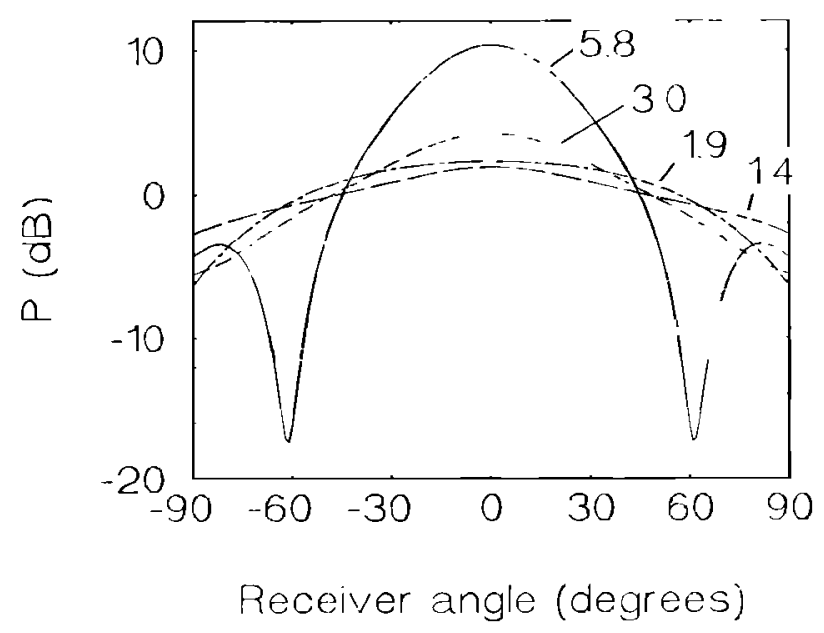

FIG. 3. Scattering from a real surface at various frequencies with standard error diffusion parameter for each frequency marked.

evaluated at a variety of frequencies and the results summed with appropriate weights depending on the frequency range where maximum diffusion is desired.

Two refinements of the standard error were found necessary:

(i) It was necessary to smooth the scattered pressure distribution before calculating the standard error. The diffracted distribution had a large number of minima and maxima which naturally produce a large standard error. Over a wider frequency range, say $1 / 3$ octave bands, these maxima and minima would smooth out and become less significant. Furthermore, theoretical predictions can produce very sharp well pronounced minima which also increase the standard error-such minima would be much less significant in practice. Smoothing of the angular distribution was done; the $I_{\theta}$ in Eq. (11) were an average over ten receivers evenly spread over ten deg. (Smoothing over a frequency range was not used because predicting at a variety of frequencies with the boundary element methods used here is computer intensive.)

(ii) A simple average of the standard errors for many frequencies allowed any poor frequency ranges for diffusion to be compensated for by other good frequency ranges. This is obviously unsatisfactory as uniform diffusion is required over the entire bandwidth. To prevent such compensation from occurring, after the standard errors were averaged over the frequencies tested, one standard error of the standard errors was added. This penalized solutions where different frequencies had greatly different diffusion characteristics. So the new diffusion parameter $\boldsymbol{\epsilon}^{\prime}$ for $\boldsymbol{n}$ frequencies each having a standard error of $\epsilon_{f i}$ was

$$
\begin{aligned}
& \epsilon^{\prime}=\bar{\epsilon}+\frac{2}{\sqrt{n(n-1)}} \sqrt{\sum_{i=1}^{n}\left(\epsilon_{f i}-\bar{\epsilon}\right)^{2},} \\
& \bar{\epsilon}=\frac{1}{n} \sum_{i=1}^{n} \epsilon_{f i} .
\end{aligned}
$$

The success of the diffusion measure is illustrated in Fig. 3. The scattered pressure distributions from a real sur- 
TABLE I. The diffuser geometries.

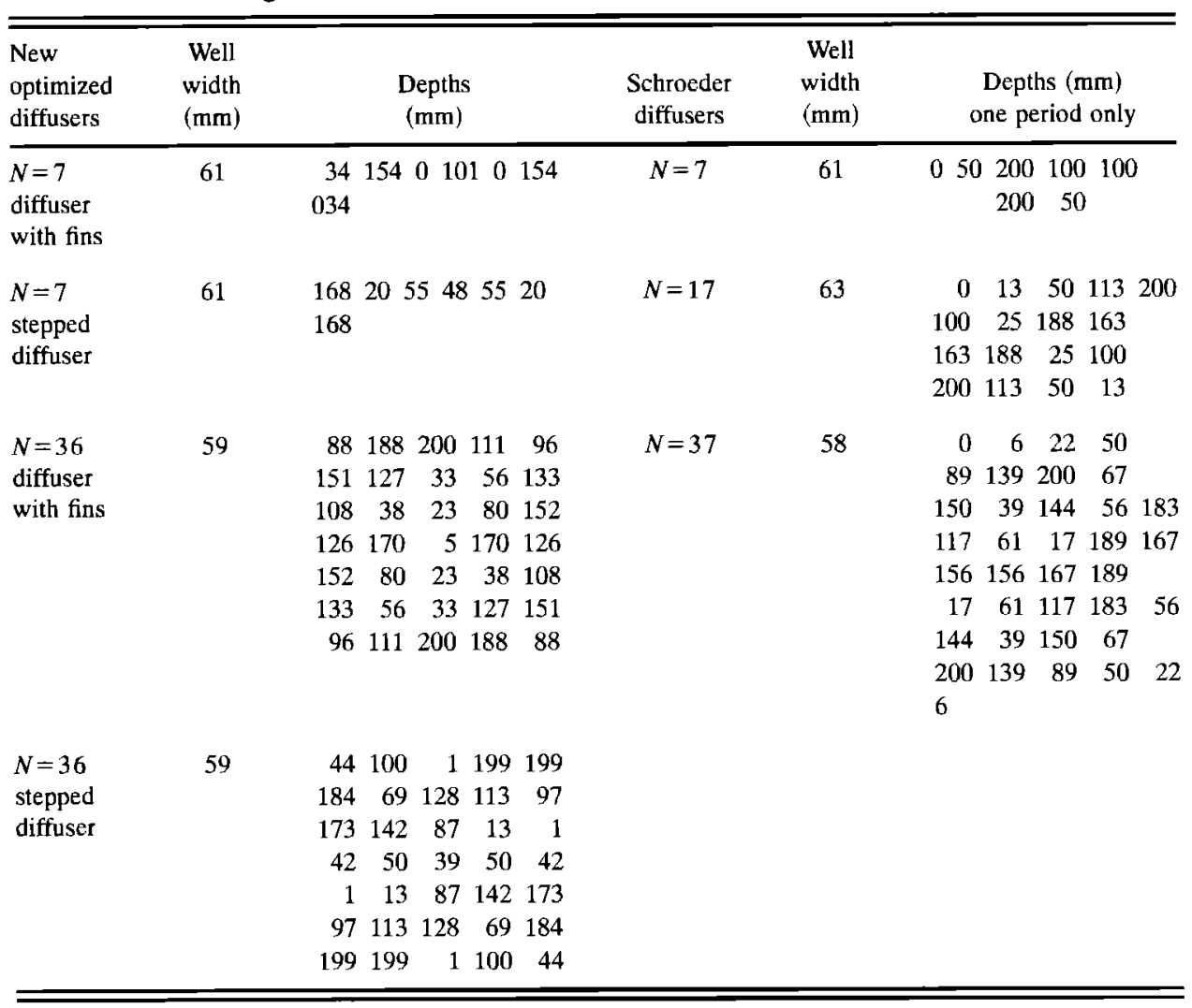

face at various frequencies are shown along with the measured standard error calculated from Eqs. (11) and (12). The standard error monitors the diffusion satisfactorily. A disadvantage in applying this diffusion parameter is that it is not known how big a difference in diffusion can be heard by listeners.

\section{B. Diffusers tested}

The diffuser designs were constrained to configurations which are used in practise and which did not have excessive absorption. Consequently, the limitations on well width and well depth suggested by D'Antonio and Konnert have been adapted; ${ }^{14}$ they have a large amount of experience of producing Schroeder diffusers. A well width of about $6 \mathrm{~cm}$ and a maximum well depth of $20 \mathrm{~cm}$ have been used.

Four different configurations have been tested:

(a) diffuser with fins, seven wells,

(b) diffuser with fins, approximately 36 wells,

(c) stepped diffuser, seven wells, and

(d) stepped diffuser, approximately 36 wells.

All these configurations have been compared to Schroeder diffusers with similar geometries. Full details of the diffuser designs can be found in Table I. This includes the well depths of the best diffusers. The source was a normal distance of $10 \mathrm{~m}$ from the diffuser, the receiver at a fixed radius of $5 \mathrm{~m}$, and the overall depth of all diffusers was 0.3 $\mathrm{m}$.

Ideally, the most accurate BEMs would have been used during all the optimization processes. Unfortunately, during optimization the scattering has to be evaluated thousands of times. This meant for diffusers over a certain width, or where there was a large number of degrees of freedom in the minimization process, the prediction times for the most accurate methods become prohibitively long. Consequently, whereas the $N=7$ diffusers could be optimized using the most accurate prediction techniques, the $N=36$ diffusers had to utilize the more approximate methods. For the final evaluation of the scattering, and for all plots given in this paper, the most accurate BEMs have been used.

This paper has been restricted to a single source from one angle of incidence. One of the primary uses of diffusers in auditoria is prevent echoes, image shift, and coloration from strong first-order reflections. In that case it seems reasonable to deal with a single angle of incidence for sound coming straight from the stage. (Ideally a small range of incidence angles should be included to allow for the variation in incidence angles due to the stage width and depth.) Although the diffusion was only optimized for one angle of incidence, the diffusers will still provide a certain amount of diffusion for higher-order reflections from other angles of incidence. There is no reason why a range of incident angles could not be used to try and obtain optimum diffusion independent of incident angle. This would, however, slow the optimization process.

A normal source was used in this project as it allowed the exploitation of symmetry, greatly reducing prediction times and halving the degrees of freedom in the minimization process. (The predictions for the Schroeder diffusers, however, were carried out with asymmetrical surfaces where appropriate.)

The work has been restricted to a single receiver radius. 


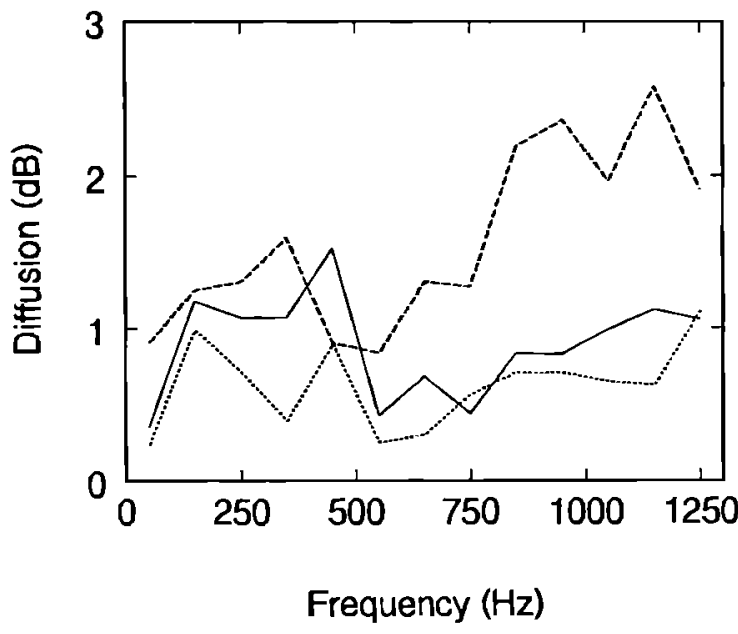

FIG. 4. Comparison of diffusion from: — optimized diffuser with fins, -- $N=7$ Schroeder diffuser, and ----- optimized stepped diffuser. Diffusion characterized by standard error parameter.

When this method is applied to a real problem, the range of receiver angles and distances encountered in the actual auditorium ought to be used.

The low-frequency performance of the diffusers has been tested (up to $1250 \mathrm{~Hz}$ ). At these frequencies, the diffusers could be used to improve the diffusion in a hall, increasing the amount of early lateral energy and so increasing the sensation of spatial impression. Producing diffusers which operate to higher frequencies might best be achieved by constructing two-way diffusers. ${ }^{14}$ For the optimization, seven randomly chosen frequencies within the bandwidth were used $(101,284,487,651,807,973$, and $1170 \mathrm{~Hz})$. This was found sufficient to get good diffusion over the bandwidth whether at or away from these optimization frequencies. The graphs displayed here are not at the optimization frequencies and so represent the worst cases of the new optimum diffusers.

\section{RESULTS}

\section{A. Diffuser with fins, seven wells}

Diffusers produced by the optimization process outperformed the $N=7$ Schroeder diffuser over a wide variety of frequencies. This is illustrated in Fig. 4. where the scattering is characterized by the standard error. In Figs. 5 and 6, the scattering at two particular frequencies are shown. (The design frequency for the Schroeder diffuser is $490 \mathrm{~Hz}^{3}$ ) The optimized diffuser's success is particularly due to the narrow nature of the Schroeder diffuser violating Schroeder's design theory. One period of $N=7$ is not an infinite repeat of a quadratic residue sequence and so the effects of the edge and the back of the diffuser are very noticeable. Consequently, for narrow diffusers dramatic improvements in the scattering can be achieved using this optimization method.

\section{B. Diffusers with fins, approximately 36 wells}

Optimization of a diffuser with 36 wells was carried out and compared to a variety of Schroeder diffusers. The Schroeder diffusers were designed to have approximately 36

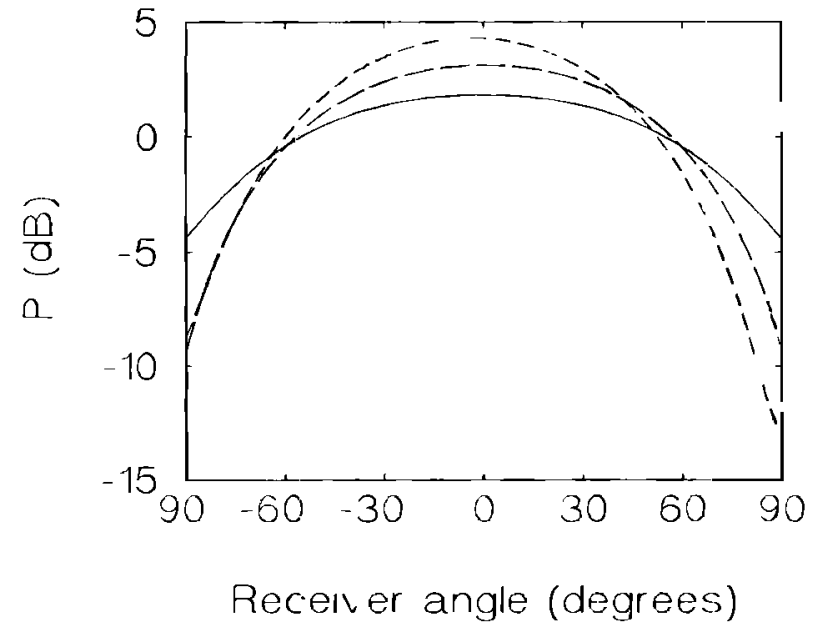

FIG. 5. Comparison of scattering from --- $N=7$ Schroeder diffuser, -.-.- optimized diffuser with fins, and - optimized stepped diffuser at $250 \mathrm{~Hz}$.

wells with the same overall width. The following were tested (periods $\times$ prime number): $5 \times 7,2 \times 17$, and $1 \times 37$. These have design frequencies of 490,808 , and $834 \mathrm{~Hz}$, respectively. In Figs. 7 and 8, comparisons between the various Schroeder diffusers and the best optimized diffuser are shown. The optimized diffuser was better than the Schroeder diffusers, although there is only a small increase in performance when compared to the $2 \times 17$ diffuser.

As the design theory behind Schroeder diffusers is only approximate, it was expected that the new optimized diffusers would create significantly better diffusion. Yet the improvement on the scattering compared to the $2 \times 17$ Schroeder diffuser is small. The failure to produce considerably better diffusion is mainly due to the fact that the scattering from Schroeder diffusers is already fairly uniform. It is difficult to produce more uniform scattering within the constraints of geometry used here. This is not, however, particularly due to the use of the quadratic residue sequence to determine the well depths. Even diffusers with randomly

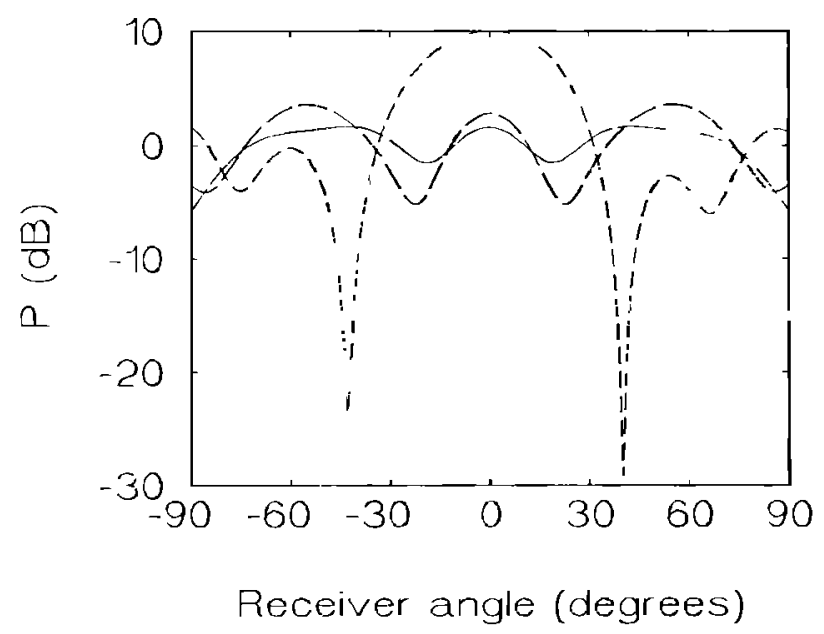

FIG. 6. Comparison of scattering from - - $N=7$ Schroeder diffuser, -.-.- optimized diffuser with fins, and _ - optimized stepped diffuser at $1050 \mathrm{~Hz}$ 


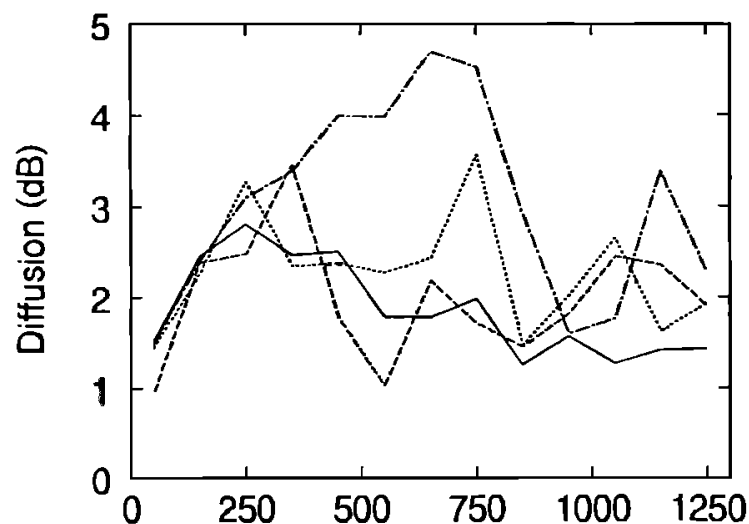

Frequency $(\mathrm{Hz})$

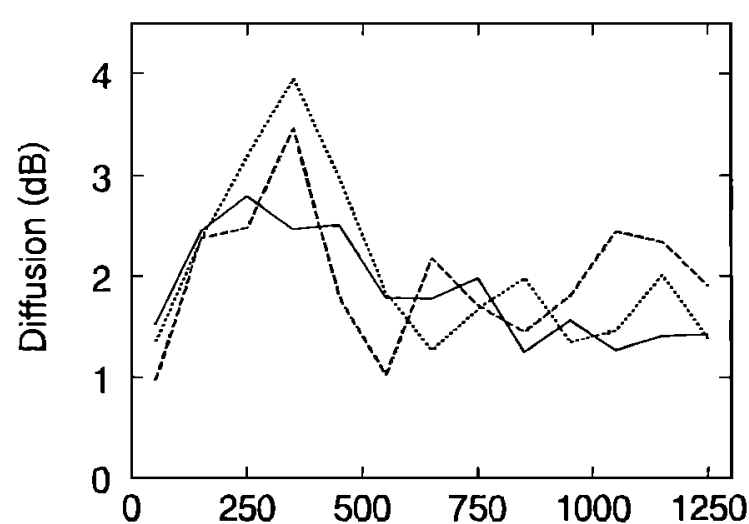

Frequency $(\mathrm{Hz})$
FIG. 7. Comparison of diffusion from various Schroeder diffusers and an optimized diffuser with fins. All diffusers with about 36 wells. -...-- $1 \times 37$ Schroeder diffuser, ---- $2 \times 17$ Schroeder diffuser; -.-.-.- $5 \times 7$ Schroeder diffuser, and —optimized diffuser with fins.

chosen depths produce reasonable diffusion. This is illustrated in Figs. 9 and 10 where the scattering from the $2 \times 17$ and optimized diffuser is compared to a diffuser with random well depths. (This diffuser has two periods of 18 randomly chosen depths.)

\section{Stepped diffusers, seven wells}

In Figs. 4-6 results from the optimization are shown. The stepped diffuser outperforms both the Schroeder diffuser and the optimum profiled diffuser at nearly all frequencies.

\section{Stepped diffuser, approximately 36 wells}

Comparisons of the scattering from the best Schroeder diffuser, the optimized diffuser with fins, and the stepped diffuser are shown in Figs. 11 and 12. As with the diffusers with seven wells, the stepped diffuser produces the best diffusion.

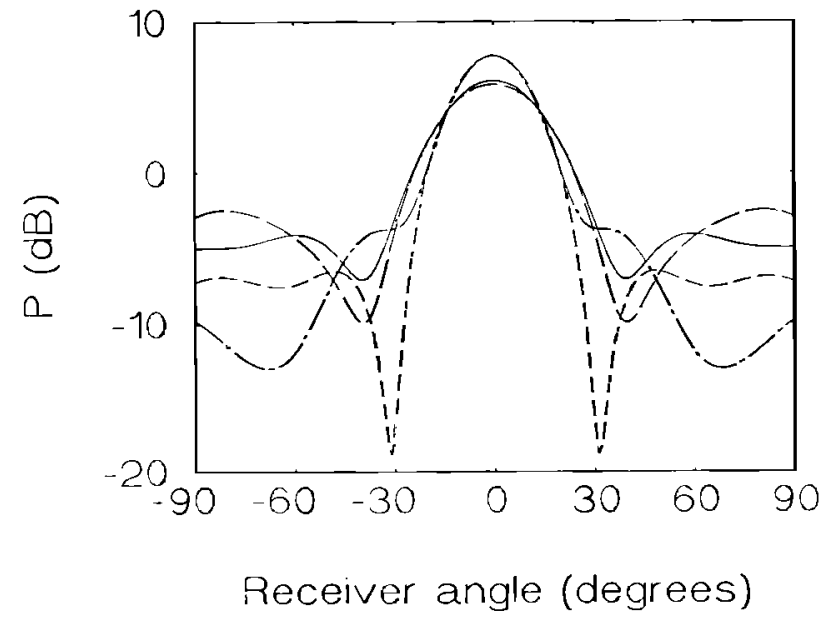

FIG. 8. Comparison of scattering from various Schroeder diffusers and an optimized diffuser with fins at $350 \mathrm{~Hz}$. All diffusers with about 36 wells. -.-.- $1 \times 37$ Schroeder diffuser, -_-- $2 \times 17$ Schroeder diffuser, ---.$5 \times 7$ Schroeder diffuser, and $\longrightarrow$ optimized diffuser with fins.
FIG. 9. Comparison of diffusion from -- $-2 \times 17$ Schroeder diffuser, an optimized diffuser with fins, and -.-.-- a diffuser with random well depths.

\section{E. Discussion of stepped diffusers}

Removing some of the constraints on geometry from Schroeder-type diffusers-by removing the fins-allows the production of better diffusion. This success shows a possible direction for further study. The methods outlined in this paper enable diffusers to be designed without rigid compliance to certain configurations. This could enable the acoustic desires of acousticians and visual requirements of architects to be better blended into an auditoria. The success of stepped diffusers was also achieved under arguably unfair criteria. Diffusers of similar absorption should be compared. This means that the stepped diffusers could have greater well depths for similar absorption as the Schroeder diffusers, and because of this, the possibility of even better diffusion.

There are two difficulties, however, with stepped diffusers. First of all, there are no simple design equations which can easily be implemented on a desktop PC as there are for Schroeder diffusers. Second, for wide diffusers or those with a large number of wells, the optimization process can be-

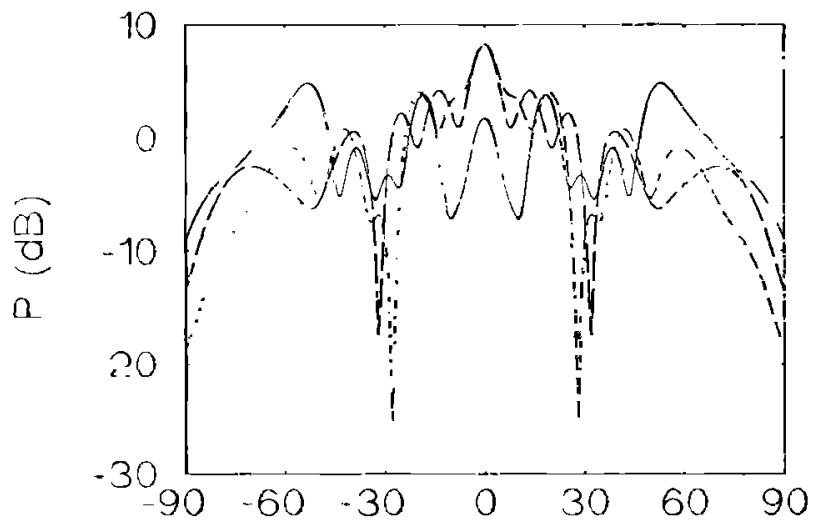

\section{Receiver angle (degrees)}

FIG. 10. Comparison of scattering from ---- $2 \times 17$ Schroeder diffuser, - an optimized diffuser with fins, and -. -. - a diffuser with random well depths $(1050 \mathrm{~Hz})$. 


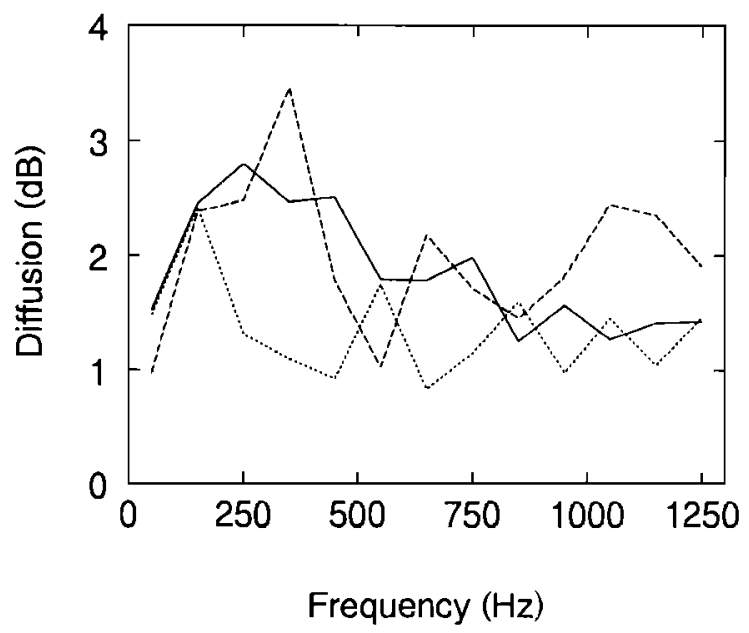

FIG. 11. Comparison of diffusion from ---- $2 \times 17$ Schroeder diffuser, - an optimized diffuser with fins, and ------ an optimized stepped diffuser.

come prohibitively long. The production of more sophisticated design methods or more powerful computers could solve this problem in the future.

\section{F. Measurements}

A reviewer of this paper carried out substantial work to confirm the results of the predicted responses; their work included measurements of the three diffusers with seven wells. These measurements confirmed the results presented above, giving extremely good agreement with the theoretical predictions and confirming the improved diffusion produced by the new optimized surfaces.

With these measurements, the reviewer also looked at the performance of the diffusers outside the domain of optimization; at higher frequencies, at other angles of incidence, for different receiver radii, and when many of the diffusers were clustered together. (The diffusers were tested for a frequency range from $300 \mathrm{~Hz}$ to $3 \mathrm{kHz}, 30^{\circ}$ and $60^{\circ}$ angles of incidence, and when two periods of the diffusers were clus-

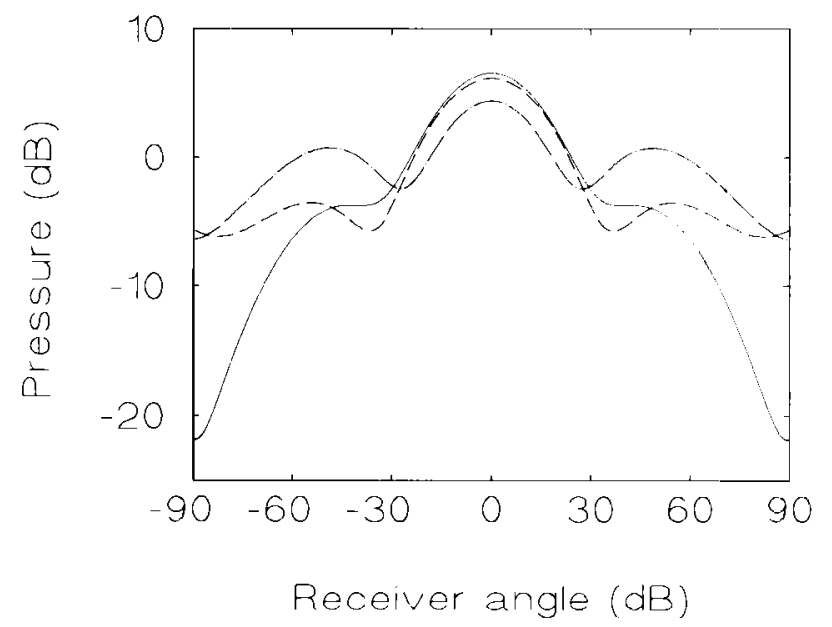

FIG. 12. Comparison of scattering from ---- $2 \times 17$ Schroeder diffuser, an optimized diffuser with fins, and $-\cdot-\cdot-$ an optimized stepped diffuser $(250 \mathrm{~Hz})$. tered together.) Outside the domain of optimization, the optimized and stepped diffusers were found to give roughly the same diffusion as the Schroeder diffusers-sometimes the new diffusers had worse diffusion, sometimes better than the Schroeder diffusers. These results demonstrated that for best results the diffusers should only be used under the conditions they have been optimized. They also usefully demonstrate that good diffusion within the optimized region has been achieved without overly penalizing the diffusers' performances elsewhere.

\section{CONCLUSIONS}

Using an iterative process the performance of profiled diffusers can be optimized. In this study the production of uniform scattering for all angles of reflection has been studied. The techniques have the potential for application to other diffusion criteria and other diffuser geometries. To monitor the degree of diffusion a measure based on standard error formulations was used and found to be successful.

Two types of optimized diffusers have been compared to Schroeder diffusers based on the quadratic residue sequence. The first type of optimized diffuser was similar to the Schroeder diffusers consisting of a series of wells of the same width, but of different depths separated by thin fins. In this case better diffusion was achieved with the optimized diffuser. The improvement was greatest for the narrow diffuser tested.

The second type of optimized diffuser was essentially a Schroeder diffuser with the fins removed. These "stepped" diffusers outperformed both Schroeder diffusers and the first type of optimized diffuser with fins. With the lack of fins, such diffusers should be cheaper to produce and be less absorbent than Schroeder diffusers.

\section{ACKNOWLEDGMENT}

The author would like to thank the staff at the Department of Applied Acoustics, University of Salford for their help with this work. 
the calculation of sound attenuation by barriers," Appl. Acoust. 31, 101117 (1990).

${ }^{10} \mathrm{~A}$. J. Burton, "The solution of Helmholtz equation in exterior domains using integral equations," National Physical Laboratory Report NAC 30, 1973.

${ }^{11}$ H. A. Schenck, "Improved integral formulation for acoustic radiation problems," J. Acoust. Soc. Am. 44, 71-100 (1968).
${ }^{12}$ W. H. Press et al., Numerical Recipes, the Art of Scientific Computing (Cambridge U.P., Cambridge, 1989), pp. 289-292.

${ }^{13}$ NAG routines library manual, Numerical Algorithms Group Ltd., Oxford, UK.

${ }^{14} \mathrm{P}$. D' Antonio and J. Konnert, "The QRD diffractal: A new one- or twodimensional fractal sound diffusor," J. Audio. Eng. Soc. 40, 117-129 (1992). 